\title{
Concept Analysis: Professional Formation of Medical Students
}

\author{
Shahram Yazdani ${ }^{1}$, Simin Esmaeilpour Zanjani ${ }^{2 *}$, \\ Leila Afshar ${ }^{3}$ and Soleiman Ahmadi ${ }^{3}$ \\ ${ }^{1}$ Department of School Medical Education Sciences, Shahid Beheshti \\ University of Medical Sciences and Health Services. Tehran, Iran. \\ ${ }^{2}$ Department of Sof Medical Education Sciences, Shahid Beheshti University of Medical \\ Sciences and Health Services. Tehran, Iran. \& Islamic Azad University, Tehran Medical Branch. \\ ${ }^{3}$ Department of School Medical Education Sciences, Shahid Beheshti \\ University of Medical Sciences and Health Services. Tehran, Iran.
}

http://dx.doi.org/10.13005/bbra/2041

(Received: 03 January 2016; accepted: 08 February 2016)

\begin{abstract}
At present, the development of concept has widely received attention and support as one of the most important approaches of exploring in order to expand and develop medical sciences. Professionalism is one of the fundamental concepts of medicine and results in the interaction between the individual and workplace, and interpersonal communications, as well as culture and value. Currently, experts in medical education believe that professional formation programs for medical students are as important as the programs on the acquisition of clinical skills and medical knowledge. This was a concept analysis study based on the Walker and Avant's approach was aimed to develop the concept of professional formation. The search terms of "professional formation", "personal and professional development", "professional identity", and "medical student" were used in the ERIC, Scopus, PubMed/MEDLINE, and Web of Science to collect the relevant studies. The retrieved studies were deeply analyzed according to the respective subject. After explaining the reason for selection, purposes, and essence of concept analysis with the specification of the main defining attributes of the intended concept, the final analytical definition of professional formation of medical students was obtained. Then, by identifying the model, borderline, related and contrary cases, antecedents and consequences of the concept, its specific dimensions were made clearer. The major challenge of the medical education is determining the most effective method of professional formation and fostering of students. In this regard, this new understanding enters a set of learning environments, designed deliberately to boost and enhance the under training professional physicians. This will lead to the transition of medical education, development in the future for performance of physicians, reduction in medical errors, and increase in the confidence of society in medical profession.
\end{abstract}

Keywords: Professional Formation, Professional Identity, Concept Analysis, Medical Education

In recent extensive investigations on the status of education in the five fields: law, medicine, the clergy, engineering, and nursing, the Carnegie Foundation concluded that "the most overlooked aspect of the education of students has been in considering the importance of their professional

\footnotetext{
* To whom all correspondence should be addressed.

E-mail: s_esmaeilpour@yahoo.com
}

identity formation; the issue is how and around what elements the mental and practical habits of students are organized"'. This foundation, with the aim of improving education, conducted a study in medical education titled "Educating Physicians: A Call for Reform of Medical Schools and Residency” in which it raised four main recommendations to stimulate the innovation and improvement of medical education: standardization and personalization of education (regulating functional 
outcomes, and allowing flexibility in learning), integration (connecting formal and experiential knowledge), the habits of research and development (focus on excellence), and the formation of professional identity (development of professional values and obligations) ${ }^{2}$. Among the 246 retrieved papers of this study showed, the most frequent citation was formation of professional identity in students ${ }^{3}$.

This issue is indeed the fundamental goal of education for all professions in general and for the medical profession in particular due to an unwritten social contract, which has resulted in considerable independence and self-regulation in this profession, in comparison with other professions. The sensitive human element in this profession requires special competencies and even internal readiness to serve people ${ }^{4}$. Professional formation in medical education is a multidimensional process in which medical students turn from lay persons to physicians ${ }^{5}$. In other words, the ultimate purpose of a medical student and medical educators is the creation and development of an identity, which is compatible with the values and competencies of medical profession ${ }^{6}$. Today, medical educationists believe that professional formation programs for students, in terms of importance, are equivalent to programs that insist on the acquisition of clinical skills and their medical knowledge ${ }^{\text {? }}$.

Studies have shown that the process of professional formation is more complex than it appears: firstly, some consider it as creating a set of attributes, attitudes, motivations, and experiences that allow the individuals to define their professional roles and achieve their personal and professional goals ${ }^{8}$; some others consider the process as "professionalism" or "practical way" 9, ${ }^{10}$ while others emphasized it under the title of "professional identity" "11. Therefore, it can be said that the issue whose situation is more complicated, is the diversity in the definition of the concept of professional formation in different scientific literatures, as well as the tendency to use different terms in explaining the concept among students, educators, policy makers, decision-makers, and researchers of medical education. The aim of the present study was to help this concept become clearer, reduce ambiguities, aid semantic integration, and increase stability in the application of this concept through the concept analysis of the process of professional formation of medical students.

\section{METHODS}

Concept analysis is a strategy used in developing the concept of professional formation and is done using several approaches that are different in terms of procedure and objective. In addition, concept analysis sometimes clarifies the concept, and sometimes offers an operational definition of the concept $\mathrm{t}^{12}$. Therefore, this study was conducted in order to define and determine the attributes and characteristics of the concept of professional formation of medical students using the Walker and Avant concept analysis model, based on the following stages:

1. Concept selection

2. Determining the purposes of analysis

3. Identifying the uses of the concept

4. Determining the defining attributes of the concept

5. Identifying a model case

6. Identifying the borderline, related, and contrary cases

7. Identifying the antecedents and consequences

8. Defining the empirical referents ${ }^{13}$.

In order to determine related articles on professional formation among the existing articles and resources, an extensive review was conducted at the beginning of the analysis, using the key words: "professional formation", "personal and professional development”, "professional identity", and "medical student" in the important databases of medical education: ERIC, Scopus, PubMed / MEDLINE, Web of Science. No time limit was considered in the search performed with a combination of related words, and all the English articles published until 2015 were included in this study. In addition, a search was performed using the Google Scholar search engine, as well as the list of the references of the articles in order to find relevant articles. In the initial search, about 1035 relevant articles were found. To exclude the unrelated sources, the first screening was performed on the title of the articles and thereafter, 692 articles were chosen. In the second stage, the abstracts of the articles were read. In this stage, 
197 articles that described the concept of professional formation or presented some attributes of the concept were selected for reading of their full text. In this step, the full texts of 45 articles were studied and only 12 articles, containing information about the professional formation process in undergraduate medical students, were selected.

\section{RESULTSAND DISCUSSION}

The concept of professional formation was selected because of its novelty and widespread use in medical literatures, educational decision-makings, policy-makings, as well as the existence of various definitions and terms serve as substitutes for this concept. The concept of professional formation of medical students and its specific dimensions and elements, as an important educational goal, are yet to be entirely apparent in medical literature. Thus, the consequence of analyzing this concept can be the origin of answering the question of this research: "What elements describe the process of professional formation of medical students?" Here, an information gap exists in the process of explaining the internal and external factors affecting the process of professional formation of medical students, especially when talking about the general medicine. This problem is more dramatic among Iranian medical schools, as the young students directly enters university from high school and do not have appropriate insight about the field they have chosen. These Iranian students choose this field of study especially due to family pressures and/or due to their imagination of the benefits that may arise and/or their gain of social prestige. This fact sometimes causes severe conflict of young and inexperienced students of their hopes and expectations, thereby resulting in a feeling of hopelessness and a search for inappropriate ways of professional self-realization in them.

According to Walker and Avant ${ }^{14}$, identifying and specifying of all applications of a concept is necessary. The main application of finding the concept of professional formation process of students as an educational goal is the new alignment and orientation of educational programs in developing this process among medical students. When the formation of professional identity (not teaching professionalism) becomes the explicit goal of medical education, new educational goals will be required and more appropriate and newer learning opportunities need to be planned around these new goals ${ }^{15,16}$. One of the applications in identifying this concept is the faculty development programs, which facilitate this process among students. The other applications of the concept will be reorienting the methods of assessing the medical students, and adopting corrective actions for them. Moreover, although it seems obvious that there are fundamental elements in professional identity that are permanent, some aspects of traditional professional identity should be changed ${ }^{11}$. As medicine and society evolve, the details of their social contract also change ${ }^{18}$, and the nature of being a good physician is continually being discussed ${ }^{18}$. An example of the need for change today, emphasizes on team care that requires a

Table 1. Empirical referents of professional formation in a medical student

Elements of professional formation

Professional attitudes and values

Familiarity with duties and responsibilities

Advanced habits of mind

Professional relationships

Perception and recognition

Acquisition of professional identity

Adherence to the professional norms

Power and hierarchy in profession

Make sense of belonging

Acquisition of tacit knowledge

Becoming familiar with professional symbols and rituals

Integration of knowledge, professional value and behavior
Method of assessment

Self-assessment, lecturer assessment

OSCE , Global Rating Scales, Mini- CEX

Portfolio

360-degree evaluation, Mini- CEX

Portfolio, OSCE

Mentor assessment

Mentor assessment and peer assessment, Mini-CEX

Mentor assessment, peer assessment, narratives

Mentor assessment, self-assessment

Mentor assessment

OSCE

Portfolio, OSCE, Mini-CEX 
change in physicians' exclusive professional identity, and accepting others' expertise ${ }^{19,20}$.

Defining characteristics of the concept are traits and attributes that distinguish the intended concept from other similar concepts, and determine its semantic space ${ }^{21}$. In order to perform this stage of concept analysis and find the defining attributes of the concept of professional formation of medical students, the selected resources were carefully studied, and phrases involving the components and/or sub constructs, goals, attributes and the method of developing the concept or revealing a certain aspect of professional formation, were specified. These reference phrases are those same initial conceptual structures that contained attributes related to the concept of professional formation of medical students and were extracted in the form of direct quotes. At this stage, the researcher tried to specify the potential definitional attributes in each reference phrase (through a gradual evolution) based on the process of abstracting her mind. Then the test of determining the necessity, antecedent and consequent was used to evaluate which of the potential definitional attributes are among the final and essential defining attributes of the intended concept.

With the specification of the main defining attributes of the intended concept, the final analytical definition of the concept of professional formation in medical students was obtained as follows:

\section{Purposed definition of professional formation}

"By adding a new dimension to the discourse of medical education, the concept of professional formation of medical students arises in order to respond to the complex social and medical challenges, maintain the social contract, prepare students for a successful role transition from student to professional and taking their professional responsibilities, obligations and roles, and deals with equipping medical students with moral development. Also, it helps in designing educational interventions in order to guide students to internalize the norms and standards, and their future professional roles, as a physician. This process is transformational and developmental, contextual, continuous, with multiple stages, adaptive, broadly and teachable and learnable, with substructures including: professional attitudes and values, becoming familiar and adapting to professional duties and responsibilities, advanced habits of mind, professional relationships, Professional perception and recognition, acquisition of professional identity, adherence to the professional norms, identification of power and hierarchy in profession, a sense of belonging to the profession, acquisition of tacit knowledge, getting familiar with professional symbols and rituals, and integration of knowledge, skills, values and behaviors. This process should occur parallel to achieving competencies aligned with professional roles and development of meta-competencies. It is necessary that the process begins stage by stage as soon as possible after the admission of students into the college, by increasing participation in their professional roles, and while exposing students to medical humanities, it should integrate the processes of experiential learning of skills along with guided reflections".

\section{Model case}

A model case is an example that has all the defining attributes of the concept. In fact, this example helps to clarify the concept further, and increases the validity of the concept ${ }^{22}$.

Ms. "A" is a final year medical student. She was very hardworking, perseverant, flexible, and interested in doing voluntary work during high school, hence she has served in one of the charity foundations for a while In the first year of her entrance into medical school, especially with the start of the clinical course, she got good grades in theoretical and practical subjects, established a very good relationship with patients, other students, professors, and hospital personnel and as such, she has always been known for these. She was always present at her apprenticeships timely and also when called upon, she performs her professional duties properly. During emergency situations, she is quick, careful and cleaver, even at midnight hours, and responds to the patient's entourage kindly and honestly while treating her patient properly. Ms. " $A$ " believes that her skills and behavior is attributed to certain things. First of all, Dr. "B" who is her role model, has always encouraged her, and has always been her guide and mentor. Thus, when necessary, he warned her about her professional errors and corrected them. Secondly, the appropriate, friendly 
and supportive atmosphere of the college and hospital, along with discussion and film criticism classes during workshops and seminars for personal development. She also considers attending conferences and sports programs on weekends along with their professors as positive experiences that considerably helped her understand the medical professional character. She considers herself committed to society, and has decided to serve people in one of the remote villages during her service plan.

\section{Creating Different Cases: Borderline, Related and} Contrary

The purpose of this comparison is to unlock the knowledge of the researcher to sensitive attributes and dimensions existing in the concept, which may be left out of sight, due to the researcher's inability to determine what he or she should be seeking for, and thus, he or she may obtain analytical blindness ${ }^{23}$.

\section{Borderline Case}

The borderline case is usually an example of the concept that has numerous defining attributes of the concept, but not all of them in itself ${ }^{21}$. In this research, the borderline concept of professional formation is the concept of professionalism, which has been increased in line with the social contract, and only focuses on performing and demonstrating professional behaviors (that is, observable behaviors that proceed from medical professional norms), but does not deal with absolute and independent principles (such as development of a value system, and understanding of oneself and/or professional socialization and acculturation) that exist in the concept of professional formation. However, this process is apparently similar only to the process of professional formation with all particularly cognitive, behavioral, symbolic, emotional, and ethical elements in which structural variables such as the university and hospital, staff, power structure and so on, have regulated the stage of behavioral socialization, and on whose development, situational variables, such as role playing, role models, peer groups, mentorship, and important emotional experiences, have dramatic influence.

\section{Related Case}

There are a variety of educational processes in the curriculum of medical schools in the world, which are very similar to the professional formation process, but they are different and do not have some defining attributes and requirements of this process. Such processes include "competency development process" and "character development process", which are in fact, part of the professional development process of students, but quite different. According to psychological theories, "professional identity development process" tries to demonstrate how to create the professional identity of a medical student. Although, there are some similarities between them, they are different in some defining attributes.

\section{Contrary Case}

The contrary case is a clear example of what the concept is not ${ }^{21}$. Putting subjects such as "medical ethics courses" in medical education curriculum with the aim of professional development, is considered a contrary case that does not include any of the defining attributes of the concept of professional formation.

\section{Antecedents}

The personal identity and attributes of a student include specific attributes (genetic status, age and gender), selective and arbitrary commitments, individual belief about him/herself, the effect of life experiences (such as culture and religion, socioeconomic status), educational and gender orientation, characteristic attributes, such as leadership, interest and seriousness, flexibility and adaptability, and capacity of progress, development and recognition, as well as the necessity of paying attention to formal and informal curriculums with the prioritization of professional values based on normative behaviors in the culture of the medical community and the management of the hidden curriculum in order to effect successful changes in medical education in the field of professional values and individual commitments, and to provide adequate reliable and supportive pedagogical space, are considered as antecedents for professional development process of medical students.

\section{Consequences}

Responding to the complex social and medical challenges and maintaining the social contract by adding a new dimension to the discourse of medical education (designing educational interventions in order to guide students to internalize the norms and standards, 
and their future professional roles as a physician and equipping them with fundamental moral principles) in order to prepare students for a successful transition from student to professional and taking their professional responsibilities, obligations and roles, are the consequents of professional formation of medical students.

The achievement of more competencies align with professional roles, as a behavioral manifestation and complement of identity in the process of becoming a physician, should not be ignored, as well as the acquisition of complex metacompetencies such as professionalism, which occur during study in medical school parallel to the formation of professional identity in medical students.

\section{CONCLUSION}

At present, the major challenge of medical education is how the most effective method of professional formation and training of students should look like. Therefore, it is required that this new understanding enters a set of learning environments, which are designed deliberately to boost and enhance the professional physicians whom are to be trained ${ }^{24}$. Since we are actively involved in the compilation and development of educational curriculums to support the professional formation of our medical students, with better understanding the fundamental elements of professional identity development process, medical socialization and factors which influence them, through this evolutionary transition more smoothly and less stressfully to achieve a new desirable identity ${ }^{25}$.

The concept of professional formation in the form of its constituent sub-structures through the methods shown in Table 1, can be assessed and has empirical referents in a medical student. But what should be considered by medical educators, professional communities, and boards offering academic degrees, is the comprehensive assessment of professional development (professional formation), which can lead to the development of the future physicians' performance, transition of medical education, reduction in medical errors, and increase in the confidence of society to medical profession.

The innovation and creation of a conceptual meaning is a logical starting point in the development of a theory and produces a set of uncertain criteria, in the case of the presence of the concept in a particular situation. The term "uncertain" means that both the definition and criteria can be revised, because it is possible that the meaning of the concept changes with the passage of time, the conduct of subsequent studies and exposure to different contexts ${ }^{26}$. Therefore, based on the analyses of the experts' views about professional formation process in the future, this study was conducted to clarify, add to and/or modify the dimensions of the concept of professional formation. Also, in future study, a theoretical model will design for the development of professional formation of undergraduate medical students.

\section{Conflict of Interest}

The authors declare that they have no conflict of interest.

\section{ACKNOWLEDGMENTS}

This research was part of a doctoral thesis in Shahid Beheshti Medical Sciences University.

\section{REFERENCES}

1. Cruess Richard L., Sylvia R. Cruess, J. Donald Boudreau, Linda Snell, and Yvonne Steinert. A Schematic Representation of the Professional Identity Formation and Socialization of Medical Students and Residents: A Guide for Medical Educators. Acad Med. 2015; 90: 718-725.

2. Irby DM1, Cooke M, O’Brien BC. Calls for reform of medical education by the Carnegie Foundation for the Advancement of Teaching: 1910 and 2010. Acad Med. 2010; 85(2):220-7. Doi: 10.1097/ACM.0b013e3181c88449.

3. O’Brien BC1, Irby DM. Enacting the Carnegie Foundation call for reform of medical school and residency. Teach Learn Med. 2013; 25 Suppl 1:S1-8. Doi: 10.1080/10401334.2013.842915

4. Hamilton, Neil. Fostering Professional Formation (Professionalism): Lessons from the Carnegie Foundation's Five Studies on Educating Professionals, 45 CREIGHTON L. REV. (forth coming 2012) (manuscript at 12) [hereinafter Hamilton, Lessons from Carnegie], available at h t t p : / / p a pers . s s r . com / s o l 3 / papers.cfm?abstract_id=1908453.

5. Holden M, Buck E, Clark M, Szauter K, Trumble 
J. Professional identity formation in medical education: The convergence of multiple domains. HEC Forum. 2012; 24:245-255.

6. Wald HS, Reis SP, Monroe AD, Borkan JM. "The loss of my elderly patient:" Interactive reflective writing to support medical students' rites of passage. Med Teach. 2010; 32:e178e184.

7. Sklar David. How Do I Figure Out What I Want to Do If I Don't Know Who I Am Supposed to Be? Academic Medicine, 2015; 90(6): 695-696.

8. Dobrow. Shoshana R. and Higgins. Monica C. Developmental networks and professional identity: a longitudinal study. Career Development International. 2005; 10(6/7: 567583.

9. Cohen J. Linking professionalism to humanism: What it means, why it matters. Acad Med. 2007; 82: 1029-1032.

10. Wilson, I., Cowin, L. S., Johnson, M. \& Young, H. Professional identity in medical students: pedagogical challenges to medical education. Teaching and Learning in Medicine: an international journal. 2013; 25 (4): 369-373.

11. Cruess RL, Cruess SR, Boudreau JD, Snell L, Steinert Y. Reframing medical education to support the development of a professional identity. Acad Med. 2014; 89: 1446-1451.

12. Powers BA, Knapp TR. Dictionary of nursing theory and research. 3rd edition. New York: Springer Publishing Company; 2006.

13. Walker LO, Avant KC. Strategies for theory construction in nursing. 4th ed. Upper Saddle River, NJ: Pearson Prentice Hall; 2005.

14. NUOPPONEN, A. 0202. Methods of concept analysis- a comparative study. LSP Journal, 0, 2-00 RIKE, C. J. \& SHARP, L. K. 0226. Assessing preservice teachers' dispositions: A critical dimension of professional preparation. Childhood Education, 62: 032-031.

15. Monrouxe Lynn V.Identity, identification and medical education: why should we care? Medical Education 2010; 44: 40-49.

16. Monrouxe LV, Rees CE, and Hu W. Differences in medical students' explicit discourses of professionalism: Acting, representing, becoming. Med Educ. 2011; 45:585-602.

17. Cruess RL, Cruess SR. Expectations and obligations: Professionalism and medicine's social contract with society. Perspect Biol Med. 2008; 51:579-598.

18. Daniels N. Just Health Care. Cambridge, England: Cambridge University Press; 2008.

19. Lingard L. What we see and don't see when we look at "competence": Notes on a god term. Adv Health Sci Educ Theory Pract. 2009; 14:625628.

20. Frost HD, Regehr G. "I am a doctor": Negotiating the discourses of standardization and diversity in professional identity construction. Acad Med. 2013; 88:1570-1577.

21. Walker, L.O., \& Avant, K.C., Concept analysis. In L.O. Walker \& K.C. Avant (Eds.), Strategies for theory construction in nursing (5th ed., pp. 157-179). Upper Saddle River, NJ: Pearson Prentice Hall, 2011.

22. McKenna H. Nursing theories and models. Rutledge essential for nurses. Taylor \& Francis e-Library. 2005. 125-259.

23. Strauss A, Corbin J. Basics of qualitative research. California: SAGE Publications; 1998.

24. Hafferty FW. Professionalism and the socialization of medical students. In: Cruess RL, Cruess SR, Steinert Y, eds. Teaching Medical Professionalism. Cambridge, UK: Cambridge University Press; 2009; 53-73.

25. Cohen JJ. Professionalism in medical education, an American perspective: from evidence to accountability. Med Educ. 2006; 40:607-617

26. Chinn PL, Kramer MK. Theory and nursing: a systematic approach. 4th edition. Mosby: St Louis; 1999. 